\title{
Neutrosophic Crisp Open Set and Neutrosophic Crisp Continuity via Neutrosophic Crisp Ideals
}

\author{
A. A. Salama ${ }^{1}$, Said Broumi ${ }^{2}$ and Florentin Smarandache ${ }^{3}$ \\ ${ }^{1}$ Department of Mathematics and Computer Science, Faculty of Sciences, Port Said University, 23 December Street, \\ Port Said 42522, Egypt. \\ ${ }^{2}$ Faculty of Arts and Humanities, Hay El Baraka Ben M'sik Casablanca B.P. 7951, Hassan II University Mohammedia- \\ Casablanca, Morocco. \\ ${ }^{3}$ Department of Mathematics, University of New Mexico,705 Gurley Avenue, Gallup, NM 87301, USA. \\ E-mail: drsalama44@gmail.com ${ }^{1}$, broumisaid78@gmail.com ${ }^{2}$, fsmarandache@gmail.com ${ }^{3}$
}

\begin{abstract}
The focus of this paper is to propose a new notion of neutrosophic crisp sets via neutrosophic crisp ideals and to study some basic operations and results in neutrosophic crisp topological spaces. Also, neutrosophic crisp L-openness and neutrosophic crisp Lcontinuity are considered as a generalizations for a crisp and fuzzy concepts. Relationships between the above new neutrosophic crisp notions and the other relevant classes are investigated. Finally, we define and study two different types of neutrosophic crisp functions.
\end{abstract}

Index Terms - Neutrosophic Crisp Set; Neutrosophic Crisp Ideals; Neutrosophic Crisp L-open Sets; Neutrosophic Crisp L- Continuity; Neutrosophic Sets.

\section{INTRODUCTION}

The fuzzy set was introduced by Zadeh [20] in 1965, where each element had a degree of membership. In 1983 the intuitionstic fuzzy set was introduced by K. Atanassov [1, 2, 3] as a generalization of fuzzy set, where besides the degree of membership and the degree of non- membership of each element. Salama et al [11] defined intuitionistic fuzzy ideal and neutrosophic ideal for a set and generalized the concept of fuzzy ideal concepts, first initiated by Sarker [19]. Smarandache [16, $17,18]$ defined the notion of neutrosophic sets, which is a generalization of Zadeh's fuzzy set and Atanassov's intuitionistic fuzzy set. Neutrosophic sets have been investigated by Salama et al. [4, 5, 6, 7, 8, 9, 10, 11, 12, $13,14,15]$. In this paper is to introduce and study some new neutrosophic crisp notions via neutrosophic crisp ideals. Also, neutrosophic crisp L-openness and neutrosophic crisp L- continuity are considered. Relationships between the above new neutrosophic crisp notions and the other relevant classes are investigated. Recently, we define and study two different types of neutrosophic crisp functions.

The paper unfolds as follows. The next section briefly introduces some definitions related to neutrosophic set theory and some terminologies of neutrosophic crisp set and neutrosophic crisp ideal. Section 3 presents neutrosophic crisp L- open and neutrosophic crisp Lclosed sets. Section 4 presents neutrosophic crisp L- continuous functions. Conclusions appear in the last section.

\section{PRELIMINARIES}

We recollect some relevant basic preliminaries, and in particular, the work of Smarandache in $[16,17,18]$, and Salama et al. $[4,5,6,7,8,9,10,11,12,13,14,15]$.

\subsection{Definitions [9].}

1) Let $\mathrm{X}$ be a non-empty fixed set. $A$ neutrosophic crisp set (NCS for short) $A$ is an object having the form $A=\left\langle A_{1}, A_{2}, A_{3}\right\rangle$ where $A_{1}, A_{2}$ and $A_{3}$ are subsets of $\mathrm{X}$ satisfying $A_{1} \cap A_{2}=\phi, A_{1} \cap A_{3}=\phi$ and $A_{2} \cap A_{3}=\phi$.

2) Let $A=\left\langle A_{1}, A_{2}, A_{3}\right\rangle$, be a neutrosophic crisp set on a set $\mathrm{X}$, then $p=\left\langle\left\{p_{1}\right\},\left\{p_{2}\right\},\left\{p_{3}\right\}\right\rangle, p_{1} \neq p_{2} \neq p_{3} \in X$ is called a neutrosophic crisp point. A neutrosophic crisp point (NCP for short) $p=\left\langle\left\{p_{1}\right\},\left\{p_{2}\right\},\left\{p_{3}\right\}\right\rangle$, is said to be belong to a neutrosophic crisp set $A=\left\langle A_{1}, A_{2}, A_{3}\right\rangle$, of X, denoted by $p \in A$, if may be defined by two types

i) Type 1: $\left\{p_{1}\right\} \subseteq A_{1},\left\{p_{2}\right\} \subseteq A_{2}$ and $\left\{p_{3}\right\} \subseteq A_{3}$,

ii) Type 2: $\left\{p_{1}\right\} \subseteq A_{1},\left\{p_{2}\right\} \supseteq A_{2}$ and $\left\{p_{3}\right\} \subseteq A_{3}$.

3) Let $X$ be non-empty set, and L a non-empty family of NCSs. We call L a neutrosophic crisp ideal (NCL for short) on $\mathrm{X}$ if

i. $A \in L$ and $B \subseteq A \Rightarrow B \in L$ [heredity],

ii. $A \in L$ and $B \in \mathrm{L} \Rightarrow A \vee B \in \mathrm{L}$ [Finite additivity].

A neutrosophic crisp ideal $\mathrm{L}$ is called a $\sigma$ neutrosophic crisp ideal if $\left\{A_{j}\right\}_{j \in \mathrm{N}} \subseteq L$, implies $\cup_{\mathrm{j} \in \mathrm{J}} \mathrm{A}_{\mathrm{j}} \in \mathrm{L}$ (countable additivity).

The smallest and largest neutrosophic crisp ideals on a 
non-empty set $\mathrm{X}$ are $\left\{\phi_{N}\right\}$ and the NSs on X. Also, $N C L_{\mathrm{f}}, \mathrm{NCL}_{\mathrm{c}}$ are denoting the neutrosophic crisp ideals (NCL for short) of neutrosophic crisp subsets having finite and countable support of $\mathrm{X}$ respectively. Moreover, if $\mathrm{A}$ is a nonempty NS in $\mathrm{X}$, then $\{B \in N C S: B \subseteq A\}$ is an NCL on $\mathrm{X}$. This is called the principal NCL of all NCSs, denoted by NCL $\langle A\rangle$.

\subsection{Proposition [9]}

Let $\left\{L_{j}: j \in J\right\}$ be any non - empty family of neutrosophic crisp ideals on a set $\mathrm{X}$. Then $\bigcap_{j \in J} L_{j}$ and $\bigcup_{j \in J} L_{j}$ are neutrosophic crisp ideals on $\mathrm{X}$, where $\curvearrowleft L_{j}=\left\langle\cap A_{j_{1}}, \cap A_{j 2}, \cup A_{j_{3}}\right\rangle$ or $\bigcap_{j \in J} L_{j}=\left\langle\bigcap_{j \in J} A_{j_{1}},{\underset{j \in J}{j \in J}}_{j \in J} A_{j_{2}},{\underset{j \in J}{J \in J}}_{A_{j 3}}^{\cup} \quad\right.$ and $\cup_{j \in J} L_{j}=\left\langle\bigcup_{j \in J} A_{j_{1}}, \cup_{j \in J} A_{j_{2}}, \bigcap_{j \in J} A_{j_{3}}\right\rangle \quad$ or $\cup_{j \in J} L_{j}=\left\langle\cup_{j \in J} A_{j_{1}}, \bigcap_{j \in J} A_{j_{2}}, \bigcap_{j \in J} A_{j_{3}}\right\rangle$.

\subsection{Proposition [9]}

A neutrosophic crisp set $A=\left\langle A_{1}, A_{2}, A_{3}\right\rangle$ in the neutrosophic crisp ideal $\mathrm{L}$ on $\mathrm{X}$ is a base of $\mathrm{L}$ iff every member of $\mathrm{L}$ is contained in $\mathrm{A}$.

\subsection{Theorem [9]}

Let $A=\left\langle A_{1}, A_{2}, A_{3}\right\rangle, \quad$ and $\quad B=\left\langle B_{1}, B_{2}, B_{3}\right\rangle, \quad$ be neutrosophic crisp subsets of X. Then $A \subseteq B$ iff $p \in A$ implies $p \in B$ for any neutrosophic crisp point $p$ in $\mathrm{X}$.

\subsection{Theorem [9]}

Let $A=\left\langle A_{1}, A_{2}, A_{3}\right\rangle$, be a neutrosophic crisp subset of X. Then $A=\cup\{p: p \in A\}$.

\subsection{Proposition [ 9]}

Let $\left\{A_{j}: j \in J\right\}$ is a family of NCSs in X. Then $\left(a_{1}\right) p=\left\langle\left\{p_{1}\right\},\left\{p_{2}\right\},\left\{p_{3}\right\}\right\rangle \in \underset{j \in J}{\cap} A_{j}$ iff $p \in A_{j}$ for each $j \in J$.

$\left(a_{2}\right) p \in \cup_{j \in J} A_{j}$ iff $\exists j \in J$ such that $p \in A_{j}$.

\subsection{Proposition [9]}

Let $A=\left\langle A_{1}, A_{2}, A_{3}\right\rangle$ and $B=\left\langle B_{1}, B_{2}, B_{3}\right\rangle$ be two neutrosophic crisp sets in $\mathrm{X}$. Then

a) $A \subseteq B$ iff for each $p$ we have $p \in A \Leftrightarrow p \in B$ and for each $p$ we have $p \in A \Rightarrow p \in B$.

b) $A=B$ iff for each $p$ we have $p \in A \Rightarrow p \in B$ and for each $p$ we have $p \in A \Leftrightarrow p \in B$.

\subsection{Proposition[9]}

Let $A=\left\langle A_{1}, A_{2}, A_{3}\right\rangle$ be a neutrosophic crisp set in $\mathrm{X}$. Then $A=\cup<\left\{p_{1}: p_{1} \in A_{1}\right\},\left\{p_{2}: p_{2} \in A_{2}\right\},\left\{p_{3}: p_{3} \in A_{3}\right\}$.

\subsection{Definition [9]}

Let $f: X \rightarrow Y$ be a function and $p$ be a neutrosophic crisp point in $\mathrm{X}$. Then the image of $p$ under $f$, denoted by $f(p)$, is defined by $f(p)=\left\langle\left\{q_{1}\right\},\left\{q_{2}\right\},\left\{q_{3}\right\}\right\rangle$, where $q_{1}=f\left(p_{1}\right), q_{2}=f\left(p_{2}\right)$ and $q_{3}=f\left(p_{3}\right)$.

It is easy to see that $f(p)$ is indeed a NCP in Y, namely $f(p)=q$, where $q=f(p)$, and it is exactly the same meaning of the image of a NCP under the function $f$.

\subsection{Definition [9]}

Let $\mathrm{p}$ be a neutrosophic crisp point of a neutrosophic crisp topological space $(X, N C \tau)$. A neutrosophic crisp neighbourhood ( NCNBD for short) of a neutrosophic crisp point $\mathrm{p}$ if there is a neutrosophic crisp open set( NCOS for short) $\mathrm{B}$ in $\mathrm{X}$ such that $p \in B \subseteq A$.

\subsection{Theorem [9]}

Let $(X, N C \tau)$ be a neutrosophic crisp topological space (NCTS for short) of $X$. Then the neutrosophic crisp set $\mathrm{A}$ of $\mathrm{X}$ is NCOS iff $\mathrm{A}$ is a NCNBD of $\mathrm{p}$ for every neutrosophic crisp set $p \in A$.

\subsection{Definition [9]}

Let $(X, \tau)$ be a neutrosophic crisp topological spaces (NCTS for short) and L be neutrosophic crisp ideal (NCL, for short) on X. Let A be any NCS of X. Then the neutrosophic crisp local function $N C A^{*}(L, \tau)$ of $\mathrm{A}$ is the union of all neutrosophic crisp point NCTS( NCP, for short) $P=\left\langle\left\{p_{1}\right\},\left\{p_{2}\right\},\left\{p_{3}\right\}\right\rangle$, such that if $U \in N((p))$ and $N A^{*}(L, \tau)=\cup\{p \in X: A \wedge U \notin L$ for every $\mathrm{U}$ nbd of $\mathrm{N}(\mathrm{P})\}$ $N C A^{*}(L, \tau)$ is called a neutrosophic crisp local function of $\mathrm{A}$ with respect to $\tau$ and $\mathrm{L}$ which it will be denoted by $N C A^{*}(L, \tau)$, or simply $N C A^{*}(\mathrm{~L})$. The 
neutrosophic crisp topology generated by $N C A^{*}(\mathrm{~L})$ in [9] we will be denoted by NC*

\subsection{Theorem [9]}

Let $(X, \tau)$ be a NCTS and $L_{1}, L_{2}$ be two neutrosophic crisp ideals on $X$. Then for any neutrosophic crisp sets $A, B$ of $\mathrm{X}$. then the following statements are verified
i) $\quad A \subseteq B \Rightarrow N C A^{*}(L, \tau) \subseteq N C B^{*}(L, \tau)$,
ii) $L_{1} \subseteq L_{2} \Rightarrow N C A^{*}\left(L_{2}, \tau\right) \subseteq N C A^{*}\left(L_{1}, \tau\right)$,
iii) $N C A^{*}=\operatorname{NCcl}\left(A^{*}\right) \subseteq N C c l(A)$,
iv) $N C A^{* *} \subseteq N C A^{*}$,
v) $N C(A \cup B)^{*}=N C A^{*} \cup N C B^{*}$,
vi) $\quad N C(A \cap B)^{*}(L) \subseteq N C A^{*}(L) \cap N C B^{*}(L)$
vii) $\ell \in L \Rightarrow N C(A \cup \ell)^{*}=N C A^{*}$
viii) $N C A^{*}(L, \tau)$ be a neutrosophic crisp closed set.

\subsection{Theorem [9]}

Let $N C \tau_{1}, N C \tau_{2}$ be two neutrosophic crisp topologies on $\mathrm{X}$. Then for any neutrosophic crisp ideal $\mathrm{L}$ on $\mathrm{X}$, $N C \tau_{1} \subseteq N C \tau_{2}$ implies $N C A^{*}\left(L, N C \tau_{2}\right) \subseteq N C A^{*}\left(N C L, N C \tau_{1}\right)$, for every $A \in L$ then $N C \tau^{*}{ }_{1} \subseteq N C \tau^{*}{ }_{2}$. A basis $N C \beta(L, \tau)$ for $N C \tau^{*}(L)$ can be described as follows:

$N C \beta(L, \tau)=\{A-B: A \in N C \tau, B \in N C L\}$. Then we have the following theorem.

\subsection{Theorem [9]}

$N C \beta(L, \tau)=\{A-B: A \in \tau, B \in L\}$ forms a basis for the generated NCTS of the NCT $(X, \tau)$ with neutrosophic crisp ideal $\mathrm{L}$ on $\mathrm{X}$.

\subsection{Theorem [9]}

Let $N C \tau_{1}, N C \tau_{2}$ be two neutrosophic crisp topologies on $\mathrm{X}$. Then for any topological neutrosophic crisp ideal $\mathrm{L} \quad$ on $\mathrm{X}, \quad N C \tau_{1} \subseteq N C \tau_{2}$ implies $N C \tau^{*}{ }_{1} \subseteq N C \tau^{*}{ }_{2}$.

\subsection{Theorem [9]}

Let $(X, \tau)$ be a NCTS and $L_{1}, L_{2}$ be two neutrosophic crisp ideals on $\mathrm{X}$. Then for any neutrosophic crisp set A in $\mathrm{X}$, we have
i) $\quad N C A^{*}\left(L_{1} \cup L_{2}, \tau\right)=N C A^{*}\left(L_{1}, N C \tau^{*}\left(L_{1}\right)\right) \cap N C A^{*}\left(L_{2}, N C \tau^{*}\left(L_{2}\right)\right)$,
ii) $N C \tau^{*}\left(L_{1} \cup L_{2}\right)=\left(N C \tau^{*}\left(L_{1}\right)\right)^{*}\left(L_{2}\right) \cap\left(N C \tau^{*}\left(L_{2}\right)\right)^{*}\left(L_{1}\right)$

\subsection{Corollary [9]}

Let $(X, \tau)$ be a NCTS with topological neutrosophic crisp ideal $\mathrm{L}$ on $\mathrm{X}$. Then

i) $N C A^{*}(L, \tau)=N C A^{*}\left(L, \tau^{*}\right)$ and $\mathrm{NC} \tau^{*}(L)=N C\left(N C \tau^{*}(L)\right)^{*}(L)$,

ii) $N C \tau^{*}\left(L_{1} \cup L_{2}\right)=\left(N C \tau^{*}\left(L_{1}\right)\right) \cup\left(N C \tau^{*}\left(L_{2}\right)\right)$.

\section{NEUTROSOPHIC CRISP L- OPEN AND NEUTROSOPHIC CRISP L- CLOSED SETS}

\section{Definition 3.1}

Given $(X, \tau)$ be a NCTS with neutrosophic crisp ideal $\mathrm{L}$ on $\mathrm{X}$, and $\mathrm{A}$ is called a neutrosophic crisp $\mathrm{L}-$ open set iff there exists $\zeta \in \tau$ such that $\mathrm{A} \subseteq \zeta \subseteq \mathrm{NCA}^{*}$.

We will denote the family of all neutrosophic crisp $\mathrm{L}-$ open sets by $\mathrm{NCLO}(\mathrm{X})$.

\section{Theorem 3.1}

Let $(\mathrm{X}, \tau)$ be a NCTS with neutrosophic crisp ideal $\mathrm{L}$, then $\mathrm{A} \in \mathrm{NCLO}(\mathrm{X})$ iff $\mathrm{A} \subseteq \mathrm{NCint}\left(\mathrm{NCA}^{*}\right)$.

\section{Proof}

Assume that $A \in \operatorname{NCLO}(X)$ then by Definition 3.1there exists $\zeta \in \tau$ such that $\mathrm{A} \subseteq \zeta \subseteq \mathrm{NCA}^{*}$. But $\operatorname{NCint}\left(\mathrm{NCA}^{*}\right) \subseteq \mathrm{NCA}^{*}$, put $\zeta=\mathrm{NCint}\left(\mathrm{NCA}^{*}\right)$. Hence $A \subseteq \mathrm{NCint}\left(\mathrm{NCA}^{*}\right)$. Conversely $\quad \mathrm{A} \subseteq \mathrm{NCint}$ $\left(\mathrm{NCA}^{*}\right) \subseteq \mathrm{NCA}^{*}$ Then there exists $\zeta=\mathrm{NCint}\left(\mathrm{NCA}^{*}\right) \in$ $\tau$. Hence $\mathrm{A} \in \mathrm{NCLO}(\mathrm{X})$.

\section{Remark 3.1}

For a NCTS $(X, \tau)$ with neutrosophic crisp ideal $\mathrm{L}$ and $\mathrm{A}$ be a neutrosophic crisp set on $\mathrm{X}$, the following holds: If $\mathrm{A} \in \mathrm{NCLO}(\mathrm{X})$ then $\mathrm{NCint}(\mathrm{A}) \subseteq \mathrm{NCA}^{*}$.

\section{Theorem 3.2}

Given $(X, \tau)$ be a NCTS with neutrosophic crisp ideal $\mathrm{L}$ on $\mathrm{X}$ and $\mathrm{A}, \mathrm{B}$ are neutrosophic crisp sets such that $\mathrm{A} \in \mathrm{NCLO}(\mathrm{X}), \mathrm{B} \in \tau$ then $\mathrm{A} \cap \mathrm{B} \in \mathrm{NCLO}(\mathrm{X})$

\section{Proof}

From the assumption $\mathrm{A} \cap \mathrm{B} \subseteq \mathrm{NCint}\left(\mathrm{NCA}^{*}\right) \cap \mathrm{B}=$ NCint $\left(\mathrm{NCA}^{*} \cap \mathrm{B}\right)$, we have $\mathrm{A} \cap \mathrm{B} \subseteq$ NCintNC(A $\cap \mathrm{B}) *$ and this complete the proof. 


\section{Corollary 3.1}

If $\left\{A_{j}\right\}_{j \in J}$ is a neutrosophic crisp L-open set in NCTS $(X, \tau)$ with neutrosophic crisp ideal L. Then $\cup\left\{A_{j}\right\}_{j \in J}$ is neutrosophic crisp L-open sets.

\section{Corollary 3.2}

For a NCTS $(X, \tau)$ with neutrosophic crisp ideal L, and neutrosophic crisp set $\mathrm{A}$ on $\mathrm{X}$ and $\mathrm{A} \in \operatorname{NCLO}(\mathrm{X})$, then $\mathrm{NCA}^{*}=\mathrm{NC}\left(\mathrm{NCintNC}\left(\mathrm{NCA} *{ }^{*}\right)\right) *$ and $\left.\mathrm{NCcl}^{*}(\mathrm{~A})\right)=\operatorname{NCint}\left(\mathrm{NCA}^{*}\right)$.

Proof: It's clear.

\section{Definition 3.2}

Given a NCTS $(X, \tau)$ with neutrosophic crisp ideal $L$ on $\mathrm{X}$ and neutrosophic crisp set $\mathrm{A}$. Then $\mathrm{A}$ is said to be:

(i) Neutrosophic crisp $\tau^{*}-$ closed (or $\mathrm{NC}^{*}$ closed) if $\mathrm{NCA}^{*} \leq \mathrm{A}$

(ii) Neutrosophic crisp L-dense - in - itself (or $\mathrm{NC}^{*}$ - dense - in - itself) if $\mathrm{A} \subseteq \mathrm{NCA}^{*}$.

(iii) Neutrosophic crisp ${ }^{*}$ - perfect if $\mathrm{A}$ is $\mathrm{NC}^{*}-$ closed and $\mathrm{NC}^{*}-$ dense - in - itself.

\section{Theorem 3.3}

Given a NCTS $(X, \tau)$ with neutrosophic crisp ideal $L$ and $\mathrm{A}$ is a neutrosophic crisp set on $\mathrm{X}$, then

(i) $\mathrm{NC}^{*}-$ closed iff $\mathrm{NCcl}^{*}(\mathrm{~A})=\mathrm{A}$.

(ii) $\mathrm{NC}^{*}$ - dense - in - itself iff $\mathrm{NCcl}^{*}(\mathrm{~A})$ $=\mathrm{NCA}^{*}$.

(iii) $\mathrm{NC}^{*}-$ perfect iff $\mathrm{NCcl}^{*}(\mathrm{~A})=\mathrm{NCA}^{*}=\mathrm{A}$.

Proof: Follows directly from the neutrosophic crisp closure operator $\mathrm{NCcl}^{*}$ for a neutrosophic crisp topology $\tau *(\mathrm{~L})$ (NC $\tau^{*}$ for short).

\section{Remark 3.2}

One can deduce that

(i) Every $\mathrm{NC}^{*}$-dense - in - itself is neutrosophic crisp dense set.

(ii) Every neutrosophic crisp closed (resp. neutrosophic crisp open) set is $\mathrm{N}^{*}$-closed (resp. NC $\tau *-$ open).

(iii) Every neutrosophic crisp L-open set is $\mathrm{NC} *-$ dense - in - itself.

\section{Corollary 3.3}

Given a NCTS $(X, \tau)$ with neutrosophic crisp ideal L on $\mathrm{X}$ and $\mathrm{A} \in \tau$ then we have: (i) If $\mathrm{A}$ is $\mathrm{NC}^{*}$-closed then $\mathrm{A}^{*} \subseteq \mathrm{NCint}(\mathrm{A})$ $\subseteq \mathrm{NCCl}(\mathrm{A})$.

(ii) If $\mathrm{A}$ is $\mathrm{NC}^{*}$-dense - in - itself then $\operatorname{Nint}(\mathrm{A})$ $\subseteq \mathrm{NCA}^{*}$.

(iii) If $\mathrm{A}$ is $\mathrm{NC}^{*}$-perfect then $\operatorname{NCint}(A)=\operatorname{NCcl}(A)=N C A *$

Proof: Obvious.

we give the relationship between neutrosophic crisp L-open set and some known neutrosophic crisp openness.

\section{Theorem 3.4}

Given a NCTS $(X, \tau)$ with neutrosophic crisp ideal L and neutrosophic crisp set $\mathrm{A}$ on $\mathrm{X}$ then the following holds:

(i) If $\mathrm{A}$ is both neutrosophic crisp $\mathrm{L}$ - open and NC*-erfect then $A$ is neutrosophic crisp open.

(ii) If $\mathrm{A}$ is both neutrosophic crisp open and NC*- dense-in - itself then A is neutrosophic crisp L-open.

Proof. Follows from the definitions.

\section{Corollary 3.4}

For a neutrosophic crisp subset A of a NCTS $(X, \tau)$ with neutrosophic crisp ideal L on X, we have:

(i) If $\mathrm{A}$ is $\mathrm{NC} *-$ closed and $\mathrm{NL}-$ open then NCint (A) $=\operatorname{NCint}\left(\mathrm{NCA}^{*}\right)$.

(ii) If $\mathrm{A}$ is $\mathrm{NC} *$-perfect and $\mathrm{NL}$-open then $\mathrm{A}=\mathrm{NC}$ int $\left(\mathrm{NCA}^{*}\right)$.

\section{Remark 3.3}

One can deduce that the intersection of two neutrosophic crisp L-open sets is neutrosophic crisp L-open.

\section{Corollary 3.5}

Given $(X, \tau)$ be a NCTS with neutrosophic crisp ideal $\mathrm{L}$ and neutrosophic crisp set $\mathrm{A}$ on $\mathrm{X}$. The following hold: If $\mathrm{L}=\left\{\mathrm{N}^{\mathrm{x}}\right\}$, then $\mathrm{NCA}^{*}(\mathrm{~L})=\phi_{N}$ and hence $\mathrm{A}$ is neutrosophic crisp L-open iff $\mathrm{A}=\phi_{N}$.

\section{Proof: It's clear.}

\section{Definition 3.5}

Given a NCTS $(X, \tau)$ with neutrosophic crisp ideal L and neutrosophic crisp set $\mathrm{A}$ then neutrosophic crisp ideal interior of $\mathrm{A}$ is defined as largest neutrosophic crisp $\mathrm{L}$-open set contained in $\mathrm{A}$, we denoted by NCL-NCint(A). 


\section{Theorem 3.5}

If $(X, \tau)$ is a NCTS with neutrosophic crisp ideal $L$ and neutrosophic crisp set $\mathrm{A}$ then

(i) $\mathrm{A} \wedge \mathrm{Nint}\left(\mathrm{NCA}^{*}\right)$ is neutrosophic crisp L-open set.

(ii) $\operatorname{NL}-\operatorname{Nint}(\mathrm{A})=0_{\mathrm{N}}$ iff $\operatorname{Nint}\left(\mathrm{NCA}^{*}\right)=0_{\mathrm{N}}$.

\section{Proof}

(i) Since NCint $\mathrm{NCA}^{*}=\mathrm{NCA}^{*} \cap \mathrm{NCint}\left(\mathrm{NCA}^{*}\right)$, then NCint $\mathrm{NCA}^{*}=\mathrm{NCA}^{*} \cap \mathrm{NCint}$ $\left(\mathrm{NCA}^{*}\right) \subseteq \mathrm{NC}\left(\mathrm{A} \cap \mathrm{NCA}^{*}\right)^{*}$. Thus $\mathrm{A} \cap \mathrm{NC}$ $\mathrm{A}^{*} \subseteq\left(\mathrm{A} \cap\left(\mathrm{A} \cap \mathrm{NCint} \mathrm{NC}\left(\mathrm{NCA}^{*}\right)\right)^{*} \subseteq\right.$ $\mathrm{NCintNC}\left(\mathrm{A} \cap \mathrm{NCint} \mathrm{NC}\left(\mathrm{NCA}^{*}\right)^{*}\right.$. Hence $\mathrm{A} \cap \mathrm{NCint}_{\mathrm{NCA}}{ }^{*} \in \mathrm{NCLO}(\mathrm{X})$.

(ii) Let $\mathrm{NCL}-\mathrm{NCint}(\mathrm{A})=\phi_{N}$, then $\mathrm{A} \cap \mathrm{A}^{*}=$ $\phi_{N}$, implies $\mathrm{NCcl}\left(\mathrm{A} \cap \mathrm{NCint}\left(\mathrm{NCA}^{*}\right)=\phi_{N}\right.$ and so $\mathrm{A} \cap$ Nint $\mathrm{A}^{*}=\phi_{N}$. Conversely

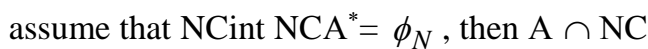
$\operatorname{int}\left(\mathrm{NC} \mathrm{A}^{*}\right)=\phi_{N}$. Hence NCL-NCint $(\mathrm{A})=$ $\phi_{N}$.

\section{Theorem 3.6}

If $(X, \tau)$ be a NCTS with neutrosophic crisp ideal L and $\mathrm{A}$ is aneutrosophic crisp set on $\mathrm{X}$, then $\mathrm{NCL}-\mathrm{NCint}(\mathrm{A})=\mathrm{A} \cap \mathrm{NCint}\left(\mathrm{NCA}^{*}\right)$.

Proof. The first implication follows from Theorem 3.4, that is $\mathrm{A} \cap \mathrm{NCA}^{*} \subseteq \mathrm{NCL}-\mathrm{NCint}(\mathrm{A})$

For the reverse inclusion, if $\zeta \in \operatorname{NCLO}(X)$ and $\zeta \subseteq$ A then $\mathrm{NC}^{*} \subseteq \mathrm{NCA}^{*}$ and hence $\mathrm{NC} \operatorname{int}\left(\mathrm{NC} \zeta^{*}\right) \subseteq$ $\operatorname{NCint}\left(\mathrm{NCA}^{*}\right)$. This implies $\zeta=\zeta \cap \operatorname{NCint}\left(\mathrm{NC} \zeta^{*}\right) \subseteq$ $\mathrm{A} \cap \mathrm{NCA}^{*}$.

Thus NCL-NCint $(\mathrm{A}) \subseteq \mathrm{A} \cap \mathrm{NCint}\left(\mathrm{NCA}^{*}\right)$

From (1) and (2) we have the result.

\section{Corollary 3.6}

For a NCTS $(X, \tau)$ with neutrosophic crisp ideal L and neutrosophic crisp set $\mathrm{A}$ on $\mathrm{X}$ then the following holds:

(i) If $\mathrm{A}$ is $\mathrm{NC} *$ - closed then $\mathrm{NL}-\mathrm{Nint}(\mathrm{A}) \subseteq \mathrm{A}$.

(ii) If $\mathrm{A}$ is $\mathrm{NC} *-$ dense - in- itself then NL $-\operatorname{Nint}(A) \subseteq A^{*}$.

(iii) If $A$ is $N C *$ - perfect set then NCL - NCint (A) $\subseteq \mathrm{NCA}^{*}$.

\section{Definition 3.6}

Given $(X, \tau)$ be a NCTS with neutrosophic crisp ideal $\mathrm{L}$ and $\zeta$ be a neutrosophic crisp set on $\mathrm{X}, \zeta$ is called neutrosophic crisp $\mathrm{L}$-closed set if its complement is neutrosophic crisp L-open set. We will denote the family of neutrosophic crisp L-closed sets by NLCC(X).

\section{Theorem 3.7}

Given $(X, \tau)$ be a NCTS with neutrosophic crisp ideal $\mathrm{L}$ and $\zeta$ be a neutrosophic crisp set on $\mathrm{X}$. $\zeta$ is neutrosophic crisp L closed, then $\mathrm{NC}(\mathrm{NCint} \zeta)^{*} \leq \zeta$.

Proof: It's clear.

\section{Theorem 3.8}

Let $(X, \tau)$ be a NCTS with neutrosophic crisp ideal L on $\mathrm{X}$ and $\zeta$ be a neutrosophic crisp set on $\mathrm{X}$ such that $\mathrm{NC}(\mathrm{NCint} \zeta)^{* \mathrm{c}}=\mathrm{NCint} \zeta^{\mathrm{c}^{*}}$ then $\zeta \in \mathrm{NLC}(\mathrm{X})$ iff $\mathrm{NC}(\mathrm{NCint} \zeta)^{*} \subseteq \zeta$.

\section{Proof}

(Necessity) Follows immedially from the above theorem (Sufficiency). Let $\mathrm{NC}\left(\mathrm{NCint} \zeta\right.$ ) ${ }^{*} \subseteq \zeta$ then $\zeta^{\mathrm{c}} \subseteq \mathrm{NC}(\mathrm{NCint} \zeta)^{* \mathrm{c}}=\mathrm{NCint}(\mathrm{NC} \zeta)^{\mathrm{c}^{*}}$. from the hypothesis. Hence $\zeta^{\mathrm{c}} \in \operatorname{NCLO}(X)$, Thus $\zeta \in \operatorname{NLCC}(X)$.

\section{Corollary 3.7}

For a NCTS $(X, \tau)$ with neutrosophic crisp ideal L on $\mathrm{X}$ the following holds:

(i) The union of neutrosophic crisp L - closed set and neutrosophic crisp closed set is neutrosophic crisp L-closed set.

(ii) The union of neutrosophic crisp $\mathrm{L}-$ closed and neutrosophic crisp L-closed is neutrosophic crisp perfect.

\section{NEUTROSOPHIC CRISP L-CONTINUOUS FUNCTIONS}

By utilizing the notion of NL - open sets, we establish in this article a class of neutrosophic crisp L- continuous function. Many characterizations and properties of this concept are investigated.

\section{Definition 4.1}

A function $f:(\mathrm{X}, \tau) \rightarrow(\mathrm{Y}, \sigma)$ with neutrosophic crisp ideal $\mathrm{L}$ on $\mathrm{X}$ is said to be neutrosophic crisp $\mathrm{L}$-continuous if for every $\zeta \in \sigma, f^{-1}(\zeta) \in \operatorname{NCLO}(\mathrm{X})$.

\section{Theorem 4.1}

For a function $f:(\mathrm{X}, \tau) \rightarrow(\mathrm{Y}, \sigma)$ with neutrosophic crisp ideal $\mathrm{L}$ on $\mathrm{X}$ the following are equivalent:

(i.) $f$ is neutrosophic crisp L-continuous. For a neutrosophic crisp point $\mathrm{p}$ in $\mathrm{X}$ and each $\zeta \in \sigma$ containing $f(\mathrm{p})$, there exists $\mathrm{A} \in \mathrm{NCLO}(\mathrm{X})$ containing $\mathrm{p}$ such that $f(\mathrm{~A}) \subseteq \sigma$. 
(ii.) For each neutrosophic crisp point $\mathrm{p}$ in $\mathrm{X}$ and $\zeta \in \sigma$ containing $f(\mathrm{p}), \quad\left(f^{-1}(\zeta)\right)^{*}$ is neutrosophic crisp nbd of $\mathrm{p}$.

(iii.) The inverse image of each neutrosophic crisp closed set in $\mathrm{Y}$ is neutrosophic crisp $\mathrm{L}$-closed.

\section{Proof}

(i) $\rightarrow$ (ii). Since $\zeta \in \sigma$ containing $f(\mathrm{p})$, then by (i), $f^{-1}(\zeta) \in \operatorname{NCLO}(\mathrm{X})$, by putting $\mathrm{A}=f^{-1}(\zeta)$ which containing $\mathrm{p}$, we have $f(\mathrm{~A}) \subseteq \sigma$

(ii) $\rightarrow$ (iii). Let $\zeta \in \sigma$ containing $f(\mathrm{p})$. Then by (ii) there exists $\mathrm{A} \in \mathrm{NCLO}(\mathrm{X})$ containing $\mathrm{p}$ such that $f(\mathrm{~A}) \leq \sigma$, so $\mathrm{p} \in \mathrm{A} \subseteq \operatorname{NCint}\left(\mathrm{NCA}^{*}\right) \leq$ NCint $\left(f^{-1}(\zeta)\right)^{*} \subseteq\left(f^{-1}(\zeta)\right)^{*}$. Hence $\left(f^{-1}(\zeta)\right)^{*}$ is neutrosophic crisp nbd of $\mathrm{p}$.

(iii) $\rightarrow$ (i) Let $\zeta \in \sigma$, since $\left(f^{-1}(\zeta)\right)$ is neutrosophic crisp nbd of any point $f^{-1}(\zeta)$, every point $\mathrm{x}_{\varepsilon} \in$ $\left(f^{-1}(\zeta)\right)^{*}$ is a neutrosophic crisp interior point of $f^{-1}(\zeta)^{*}$. Then $f^{-1}(\zeta) \subseteq$ NCint NC $\left(f^{-1}(\zeta)\right)^{*}$ and hence $f$ is neutrosophic crisp L-continuous

(i) $\rightarrow$ (iv) Let $\xi \in$ y be a neutrosophic crisp closed set. Then $\xi^{\mathrm{c}}$ is neutrosophic crisp open set, by $f^{-1}\left(\xi^{c}\right)=\left(f^{-1}(\xi)\right)^{c} \in \operatorname{NCLO}(\mathrm{X})$. Thus $f^{-1}(\xi)$ is neutrosophic crisp L-closed set.

The following theorem establish the relationship between neutrosophic crisp L-continuous and neutrosophic crisp continuous by using the previous neutrosophic crisp notions.

\section{Theorem 4.2}

Given $f:(\mathrm{X}, \tau) \rightarrow(\mathrm{Y}, \sigma)$ is a function with a neutrosophic crisp ideal $\mathrm{L}$ on $\mathrm{X}$ then we have. If $f$ is neutrosophic crisp L- continuous of each neutrosophic crisp*-perfect set in $\mathrm{X}$, then $f$ is neutrosophic crisp continuous.

\section{Proof: Obvious.}

\section{Corollary 4.1}

Given a function $f:(\mathrm{X}, \tau) \rightarrow(\mathrm{Y}, \sigma)$ and each member of $\mathrm{X}$ is neutrosophic crisp $\mathrm{NC}^{*}$-dense - in - itself. Then we have every neutrosophic crisp continuous function is neutrosophic crisp NCL-continuous.

Proof: It's clear.

We define and study two different types of neutrosophic crisp functions.

\section{Definition 4.2}

A function $f:(X, \tau) \rightarrow(Y, \sigma)$ with neutrosophic crisp ideal $\mathrm{L}$ on $\mathrm{Y}$ is called neutrosophic crisp L-open (resp. neutrosophic crisp NCL- closed), if for each $A \in \tau$ (resp. $\mathrm{A}$ is neutrosophic crisp closed in $\mathrm{X})$, $f(A) \in N C L O(Y)$ (resp. $f(A)$ is NCL-closed).

\section{Theorem 4.3}

Let a function $f:(X, \tau) \rightarrow(Y, \sigma)$ with neutrosophic crisp ideal $\mathrm{L}$ on $\mathrm{Y}$. Then the following are equivalent:

(i.) $f$ is neutrosophic crisp L-open.

(ii.) For each $\mathrm{p}$ in $\mathrm{X}$ and each neutrosophic crisp ncnbd A of $\mathrm{p}$, there exists a neutrosophic crisp Lopen set $B \in I^{Y}$ containing $f(p)$ such that $B \subseteq f(A)$

Proof: Obvious.

\section{Theorem 4.4}

A neutrosophic crisp function $f:(X, \tau) \rightarrow(Y, \sigma)$ with neutrosophic crisp ideal $\mathrm{L}$ on $\mathrm{Y}$ be a neutrosophic crisp L-open (resp.neutrosophic crisp L-closed), if A in $\mathrm{Y}$ and $\mathrm{B}$ in $\mathrm{X}$ is a neutrosophic crisp closed (resp. neutrosophic crisp open ) set $\mathrm{C}$ in $\mathrm{Y}$ containing A such that $f^{-1}(C) \subseteq B$

\section{Proof}

Assume that $A=1_{Y}-\left(f\left(1_{X}-B\right)\right)$, since $f^{-1}(C) \leq B$ and $A \leq C$ then $\mathrm{C}$ is neutrosophic crisp L-closed and $f^{-1}(C)=1_{X}-f^{-1}\left(f\left(1_{X}-A\right)\right) \leq B$.

\section{Theorem 4.5}

If a function $f:(X, \tau) \rightarrow(Y, \sigma)$ with neutrosophic crisp ideal $\mathrm{L}$ on $\mathrm{Y}$ is a neutrosophic crisp L-open, then $f^{-1} N C(N C \operatorname{int}(A))^{*} \leq N C\left(f^{-1}(A)\right)^{*}$ such that $f^{-1}(A)$ is neutrosophic crisp*-dense-in-itself and $\mathrm{A}$ in $\mathrm{Y}$.

\section{Proof}

Since $\mathrm{A}$ in $\mathrm{Y}, N C\left(f^{-1}(A)\right)^{*}$ is neutrosophic crisp closed in $\mathrm{X}$ containing $f^{-1}(A), \boldsymbol{f}$ is neutrosophic crisp L-open then by using Theorem 4.4 there is a neutrosophic crisp L-closed set $A \subseteq B$ suchthat, $\left(f^{-1}(A)\right)^{*} \supseteq f^{-1}(B) \geq f^{-1} N C(\operatorname{int}(B))^{*} \supseteq f^{-1} N C(N C \operatorname{int}(\mu))^{*}$. 


\section{Corollary 4.2}

For any bijective function $f:(X, \tau) \rightarrow(Y, \sigma)$ with neutrosophic crisp ideal $\mathrm{L}$ on $\mathrm{Y}$, the following are equivalent:

(i.) $f^{-1}:(Y, \sigma) \rightarrow(X, \tau)$ is neutrosophic crisp Lcontinuous.

(ii.) $f$ is neutrosophic crisp L-open.

(iii.) $f$ is neutrosophic crisp L-closed.

Proof: Follows directly from Definitions.

\section{CONCLUSION}

In our work, we have put forward some new concepts of neutrosophic crisp open set and neutrosophic crisp continuity via neutrosophic crisp ideals. Some related properties have been established with example. It 's hoped that our work will enhance this study in neutrosophic set theory.

\section{REFERENCES}

[1] K. Atanassov, intuitionistic fuzzy sets, in V.Sgurev, ed., Vii ITKRS Session, Sofia (June 1983 central Sci. and Techn. Library, Bulg. Academy of Sciences (1984).

[2] K. Atanassov, intuitionistic fuzzy sets, Fuzzy Sets and Systems 20, (1986),pp. 87-96.

[3] K. Atanassov, Review and new result on intuitionistic fuzzy sets, preprint IM-MFAIS-Sofia, (1988), pp.1-88.

[4] S. A. Alblowi, A.A.Salama and Mohmed Eisa, New Concepts of Neutrosophic Sets, International Journal of Mathematics and Computer Applications Research (IJMCAR),Vol. 4, Issue 1, (2014),pp. 59-66.

[5] I. Hanafy, A.A. Salama and K. Mahfouz, Correlation of Neutrosophic Data, International Refereed Journal of Engineering and Science (IRJES), Vol.(1), Issue 2 .(2012), pp.33-39.

[6] I.M. Hanafy, A.A. Salama and K.M. Mahfouz,," Neutrosophic Classical Events and Its Probability" International Journal of Mathematics and Computer Applications Research(IJMCAR) Vol.(3),Issue 1, (2013), pp.171-178.

[7] A. A. Salama and S.A. Alblowi, "Generalized Neutrosophic Set and Generalized Neutrosophic Spaces,"Journal Computer Sci. Engineering, Vol. (2) No. (7) (2012),pp.129-132.

[8] A. A. Salama and S. A. Alblowi, Neutrosophic Set and Neutrosophic Topological Spaces, ISOR J. Mathematics, Vol.(3), Issue(3), (2012), pp-31-35.

[9] A. A. Salama, "Neutrosophic Crisp Point \& Neutrosophic Crisp Ideals", Neutrosophic Sets and Systems, Vol.1, No. 1, (2013), pp. 50-54.

[10] A. A. Salama and F. Smarandache, "Filters via Neutrosophic Crisp Sets", Neutrosophic Sets and Systems, Vol.1, No. 1, (2013), pp. 34-38.

[11] A.A. Salama and S.A. Alblowi, Intuitionistic Fuzzy Ideals Spaces, Advances in Fuzzy Mathematics, Vol.(7), Number 1, (2012) pp. 51- 60.

[12] A.A. Salama, and H.Elagamy, "Neutrosophic Filters" International Journal of Computer Science Engineering and Information Technology Reseearch (IJCSEITR),
Vol.3, Issue(1),Mar 2013,(2013), pp. 307-312.

[13] A. A. Salama, F.Smarandache and Valeri Kroumov "Neutrosophic crisp Sets \& Neutrosophic crisp Topological Spaces" Bulletin of the Research Institute of Technology (Okayama University of Science, Japan), in January-February (2014). (Accepted).

[14] A. A. Salama, Mohamed Eisa and M. M . Abdelmoghny, "Neutrosophic Relations Database" International Journal of Information Science and Intelligent System, 3(1) (2014).

[15] A. A. Salama, Florentin Smarandache and S. A. ALblowi, New Neutrosophic Crisp Topological Concepts, Neutrosophic Sets and Systems, Vol.2, No. 1, (2014).

[16] Florentin Smarandache, Neutrosophy and Neutrosophic Logic, First International Conference on Neutrosophy , Neutrosophic Logic, Set, Probability, and Statistics University of New Mexico, Gallup, NM 87301, USA(2002).

[17] F. Smarandache, A Unifying Field in Logics: Neutrosophic Logic. Neutrosophy, Neutrosophic crisp Set, Neutrosophic Probability. American Research Press, Rehoboth, NM, (1999).

[18] F. Smarandache, Neutrosophic set, a generialization of the intuituionistics fuzzy sets, Inter. J. Pure Appl. Math., 24 (2005), pp.287 - 297.

[19] Debasis Sarker, Fuzzy ideal theory, Fuzzy local function and generated fuzzy topology, Fuzzy Sets and Systems 87, (1997),pp.117- 123.

[20] L.A. Zadeh, Fuzzy Sets, Inform and Control 8, ,(1965),pp.338-353.

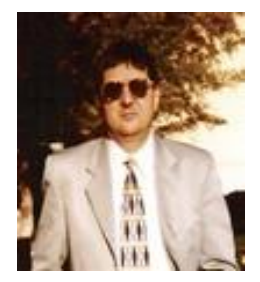

Dr. Florentin Smarandache is a Professor of Mathematics at the University of New Mexico in USA. He published over 75 books and 200 articles and notes in mathematics, physics, philosophy, psychology, rebus, literature. In mathematics his research is in number theory, non-Euclidean geometry, synthetic geometry, algebraic structures, statistics, neutrosophic logic and set (generalizations of fuzzy logic and set respectively), neutrosophic probability (generalization of classical and imprecise probability).Also, small contributions to nuclear and particle physics, information fusion, neutrosophy (a generalization of dialectics), law of sensations and stimuli, etc. He got the 2010 Telesio-Galilei Academy of Science Gold Medal, Adjunct Professor (equivalent to Doctor Honoris Causa) of Beijing Jiaotong University in 2011, and 2011 Romanian Academy Award for Technical Science (the highest in the country). Dr. W. B. Vasantha Kandasamy and Dr.Florentin Smarandache got the 2012 and 2011 New Mexico-Arizona Book Award for Algebraic Structures.

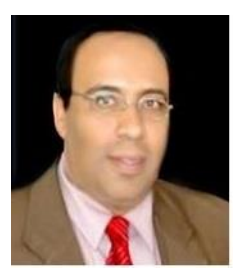

Dr. A. A. Salama (Ahmed Salama) Doctor and Lecturer in Mathematics and Computer Sciences Department in Faculty of Science in Port Said University. Associate Professor of Pure Mathematics \& Computer Science in Baha College of Sciences, Saudi Arabia. Obtained Doctoral degree in 2001 in Pure Mathematics. 3. He published over 100 articles and notes in mathematics, computer science and Statistics. 
- The first Arab to use the Neutrosophic concepts in these areas (computer Sci., Math, Statistics and Topology).

- A member of its Editorial Board to International Journal's Neutrosophic Set and Systems (USA).

- He published over 100 articles and notes in mathematics, computer science and Statistics.

- Reviewers of The Book MARIUS COMAN THE MATH ENCYCLOPEDIA OF SMARANDACHE TYPE NOTIONS. I. NUMBER THEORY Educational Publishing , 2013 by Marius Coman Education Publishing USA.

- A member of Editorial Board SMARANDACHE NOTIONS Journal's Vol.iii, ii, i.USA.

- Manager of the Quality Assurance Unit, Port Said Faculty of Science.

- Head of the Committee of Training and Community Service, Al-Baha Private College of Science.

- Educational Supervisor of Mathematics in the Zahraa Islamic for Language Schools, Mansoura for six years.

- Secretary-general of Topology Conference held in the Suez Canal University, 2007.

- Staff Member in the Higher Institute Tebah for Computer and Administrative Sciences, Maadi, Cairo, Egypt.
- Head of the Board of Al-Haram Educational Periodical published in London.

- American Diploma Delegate, American Eagles Schools. 2005-2006.

- Main research points currently are Neutrosophic Mathematics, Computer Sciences and Statistics.

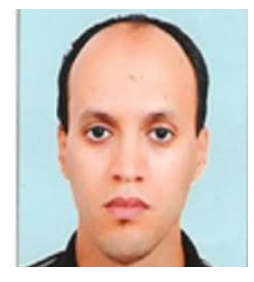

Said Broumi worked in Hassan II university Mohammedia- Casablanca as an administrator. $\mathrm{He}$ worked in University for six years. He received his M. Sc in Industrial Automatic from Hassan II University Ain chokCasablanca. His research concentrates on soft set theory, fuzzy theory, intuitionistic fuzzy theory, neutrosophic theory, control systems. He has published 15 articles in international journals.

How to cite this paper: A. A. Salama, Said Broumi, Florentin Smarandache,"Neutrosophic Crisp Open Set and Neutrosophic Crisp Continuity via Neutrosophic Crisp Ideals", IJIEEB, vol.6, no.3, pp.1-8, 2014. DOI: 10.5815/ijieeb.2014.03.01 